\title{
Análise de Antenas de Microfita sobre Substratos Cilíndricos Circulares com Cobertura Dielétrica
}

Franklin da Costa Silva, Sérgio B. de Assis Fonseca, Antonio José M. Soares e Attílio José Giarola

\begin{abstract}
Neste trabalho exploram-se as potencialidades das funções de Green diádicas e do método dos momentos, nas soluçōes de problemas de antenas de microfita, sobre substratos cilíndricos circulares. Determinam-se as funções de Green diádicas do tipo elétrico, para um meio formado por quatro camadas cilíndricas concêntricas, sendo a camada central um condutor perfeito, e, equaçōes integrais, que determinam o comportamento dos campos eletromagnéticos, são obtidas. Analisa-se o efeito da cobertura dielétrica, na freqüência de ressonância de uma antena de microfita, com elemento ressoador "retangular", alimentada diretamente por uma linha de microfita e montada sobre substrato cilíndrico circular. Os resultados indicam que o efeito da cobertura dielétrica é substancial quando o valor da constante dielétrica relativa e da espessura da cobertura são aumentados, de forma que, nos projetos de antenas de microfita, este efeito tem que ser cuidadosamente analisado.
\end{abstract}

\section{Introdução}

A análise de problemas eletromagnéticos, em meios de várias camadas, guias de onda, etc, recebeu notável impulso após as publicaçōes dos trabalhos de Tai [1]-[3], tratando da aplicaçāo das funçōes de Green diádicas em teoria eletromagnética. Uma vez que as antenas de microfita sāo montadas em meios com multicamadas, é conveniente no estudo destas, a aplicaçāo da técnica das funçōes de Green diádicas.

Sendo a característica mais importante das antenas de microfita, o fato que elas se adaptam a superfícies de várias formas, o estudo do desempenho destas antenas sobre substratos cilíndricos é relevante, uma vez que estes substratos são comuns nos projetos de circuitos de microfita.

F.C. Silva, S.B.A. Fonseca e A.J.M. Soares são Professores do Departamento de Engenharia Elétrica da UnB, 70910, Brasília, DF.

A.J. Giarola é Professor do Departamento de Microonda e Óptica, FEE, UNICAMP, 13081, Campinas, SP. 
Um grande número de trabalhos foi desenvolvido visando uma compreensāo detalhada das linhas de microfita sobre substratos planos. O estudo de linhas de microfita sobre substratos cilíndricos circulares é de particular importância para uso em projetos de antenas de microfita sobre estes substratos. Também, pouco existe disponível com relaçāo a este assunto na literatura científica. Uma análise teórica, usando-se modelo dinâmico para elementos de microfita sobre substratos cilindrícos, foi desenvolvida por Nakatani e Alexipoulos [4], sendo que, foram considerados apenas substratos com camada singela. Entretanto, em muitas aplicaçōes, as linhas de microfita sāo projetadas com cobertura dielétrica. O método dos momentos [5] tem sido amplamente utilizado para computar a impedância de entrada de antenas de microfita, alimentadas por linhas de microfita, sobre substratos planos [6]-[8].

$\mathrm{Na}$ Seçāo 2, determinam-se as funçōes de Green diádicas do tipo elétrico, para um meio constituido de quatro camadas cilíndricas concêntricas, sendo a camada central um condutor perfeito. Na Seção 3, soluciona-se o problema de uma linha de microfita infinita, montada sobre substrato cilíndrico circular, calculando sua constante de propagaçāo, através da aplicação das funçōes de Green diádicas. Resultados numéricos são apresentados, dando ênfase ao efeito da cobertura dielétrica. $\mathrm{Na}$ Seção 4, equaciona-se oproblema de uma antena de microfita "retangular", alimentada diretamente por uma linha de microfita, estando o conjunto linha-antena montado sobre substrato cilíndrico circular, com cobertura dielétrica. Uma equaçāo integral é obtida, usando-se a técnica das funçōes de Green diádicas. A seguir, resolve-se esta equaçāo integral, para as correntes introduzidas na superfície do conjunto, através da aplicação do método dos momentos. Apresentam-se, entāo, resultados numéricos mostrando o efeito da cobertura dielétrica na freqüência de ressonância da antena. $\mathrm{Na}$ análise, é assumida uma dependência com o tempo do tipo exp (-iwt).

\section{Funçōes de Green Diádicas para Meios com Quatro Camadas Cilíndricas}

O conhecimento da funçāo de Green diádica para o espaço livre, do tipo elétrico ou magnético, permite a obtenção das funçōes de Green diádicas para outros meios através da aplicaçăo do método da superposiçāo por espalhamento [1] e [9]-[11]. A funçāo de Green diádica do tipo elétrico para o espaço livre satisfaz a seguinte equaçáo de onda diádica [1]

$$
\nabla \times \nabla \times \overline{\bar{G}}_{e 0}\left(\bar{R} / \overline{R^{\prime}}\right)-k^{2} \overline{\bar{G}}_{e 0}\left(\bar{R} / \overline{R^{\prime}}\right)=\overline{\bar{I}} \delta\left(\bar{R}-\overline{R^{\prime}}\right)
$$


Esta equação pode ser resolvida pelo método de Ohm-Rayleigh, resultando, em coordenadas cilíndricas circulares [1]

$$
\begin{aligned}
& \overline{\bar{G}}_{e o}\left(\bar{R} / \bar{R}^{\prime}\right)=-\frac{1}{k_{2}} \delta\left(\bar{R}-\overline{R^{\prime}}\right) \hat{r} \hat{r}+\frac{i}{8 \pi} \int_{\infty}^{-\infty} d h \sum_{\infty}^{n=0}\left(2-\delta_{0}\right) / \lambda^{2}
\end{aligned}
$$

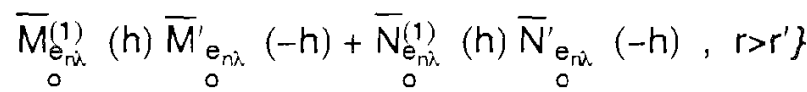

$$
\begin{aligned}
& \bar{M}_{e_{r \lambda}}(h) \bar{M}_{0}^{\prime}(1)(-h)+\bar{N}_{e_{n \lambda}}^{0}(h) \bar{N}_{0}^{(1)}(-h), r<r^{\prime}
\end{aligned}
$$

onde $\lambda^{2}=k^{2}-h^{2}, k=\omega\left(\mu_{0} \varepsilon_{0}\right)^{1 / 2}$ é o número de onda do espaço livre e $\delta_{0}$ denota a funçáo delta de Kronecker, ou seja

$$
\delta_{0}= \begin{cases}1, & n=0 \\ 0, & n \neq 0\end{cases}
$$

Adota-se, em (2), a seguinte notação simplificada

$$
\bar{F}_{e} \bar{F}_{\substack{o \\ o}}^{\prime}=\bar{F}_{e} \bar{F}_{e}^{\prime}+\bar{F}_{o} \bar{F}_{o}^{\prime}
$$

As expressōes para as funçōes $\bar{M}$ e $\bar{N}$ sāo dadas por [1]

$$
\begin{aligned}
& \bar{M}_{\substack{e_{n \lambda} \\
0}}(h)=\left[\mp \frac{n}{r} J_{n}(\lambda r) \frac{\operatorname{sen}}{\cos }(n \varphi) \hat{r}\right. \\
& \left.-\frac{\partial}{\partial} r J_{n}(\lambda r) \frac{\cos }{\operatorname{sen}}(n \varphi) \hat{\varphi}\right] \exp (i n z)
\end{aligned}
$$

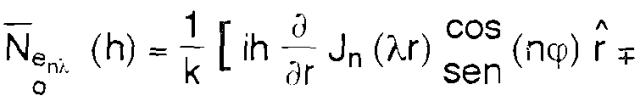

Revista da Sociedade Brasileira de Telecomunicaçôes

Volume 5. $N^{\mathrm{N}} 2$, dezembro de 1990

27 
$\left.\mp(i h n / r) J_{n}(\lambda r) \underset{\cos }{\operatorname{sen}}(n \varphi) \hat{\varphi}+\lambda^{2} J_{n}(\lambda r) \operatorname{sen}_{\cos }^{\cos }(n \varphi) \hat{z}\right] \exp (i h z)$

As funçōes $\overline{M^{\prime}}$ e $\overline{N^{\prime}}$ sāo as funçōes $\bar{M}$ e $\bar{N}$ definidas com respeito às coordenadas da fonte $\left(r^{\prime}, \phi^{\prime}, z^{\prime}\right)$, enquanto que as funçōes $\bar{M}^{(1)}$ e $\bar{N}^{(1)}$ são expressas em termos da funçăo de Hankel de primeira espécie e n-ésima ordem, substituindo-se $J_{n}(\lambda r)$ por $H_{n}^{(1)}(\lambda r)$ em (5) e (6).

Um meio constituído de quatro camadas cilíndricas concêntricas, infinitas na direção $\mathbf{Z}$, com uma fonte de corrente elétrica arbitrária localizada na regiāo 2 , tem uma seçāo transversal como mostrada na Fig. 1. Definem-se as regiōes de forma que

Regiāo 1: $k_{1}=\omega\left(\mu_{1} \varepsilon_{1}\right)^{1 / 2}$ e $\lambda_{f}^{2}=k_{1}^{2}-h^{2}$

Regiāo 2: $k_{2}=\omega\left(\mu_{2} \varepsilon_{2}\right)^{1 / 2}$ e $\lambda_{2}^{2}=k_{2}^{2}-h^{2}$

Regiāo 3: $k_{3}=\omega\left(\mu_{3} \varepsilon_{3}\right)^{1 / 2}$ e $\lambda_{3}^{2}=k_{3}^{2}-h^{2}$

Regiāo 4: $\sigma_{4}=\infty$

onde $\mu_{i}$ e $\varepsilon_{i}$ denotam, respectivamente, a permeabilidade e a permissividade da regiáo $i$, e s é a condutividade elétrica da regiāo 4, assumida infinita.

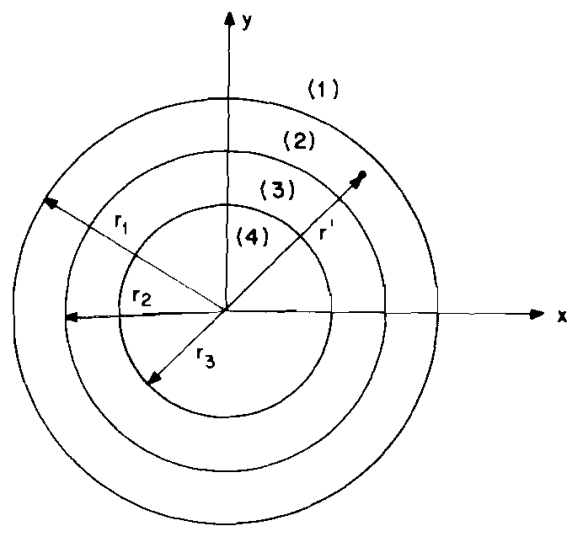

Figura 1. Seçāo transversal de um meio constituído de quatro camadas cilíndricas concêntricas. 
Como a fonte se localiza na regiāo 2, tem-se as seguintes funçōes de Green diádicas do tipo elétrico [9]:

\section{Regiāo 1:}

$$
\begin{aligned}
& \overline{\bar{G}}(1,2)\left(\bar{R} / \bar{R}^{\prime}\right)=\frac{i}{8 \pi} \int_{-\infty}^{+\infty} d h \sum_{n=0}^{\infty}\left(2-\delta_{0}\right) / \lambda_{2}^{2}
\end{aligned}
$$

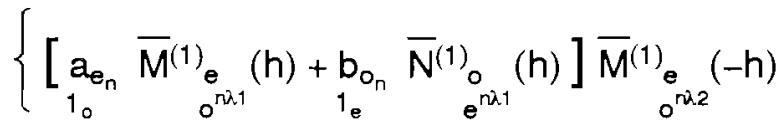

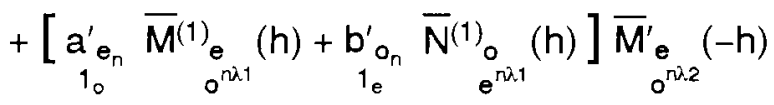

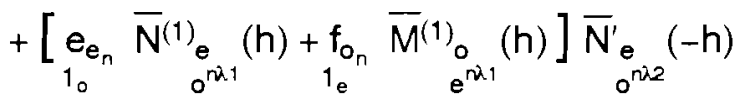

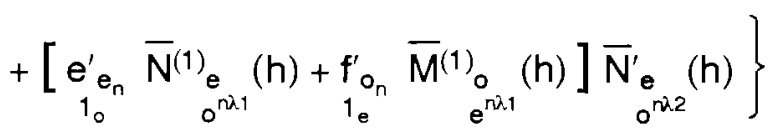

Regiāo 2:

$$
\begin{aligned}
& \overline{\bar{G}}(2,2)\left(\bar{R} / \bar{R}^{\prime}\right)=-\delta\left(\bar{R}-\bar{R}^{\prime}\right) \hat{r} \hat{r} / k_{2}^{2}+\frac{i}{8 \pi} \int_{-\infty}^{+\infty} d h \sum_{n=0}^{\infty}\left(2-\delta_{0}\right) / \lambda \lambda_{2}^{2}
\end{aligned}
$$

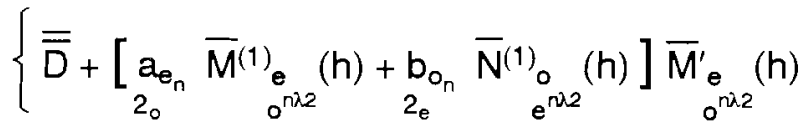

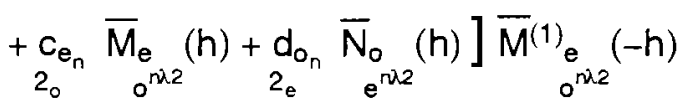




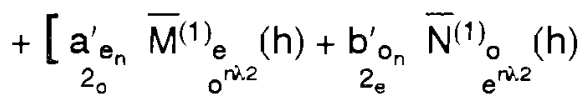

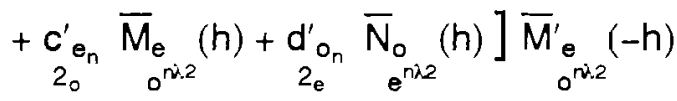

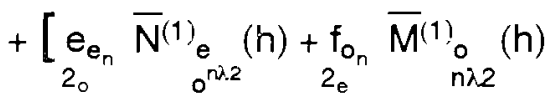

$$
\begin{aligned}
& \left.+\underset{2_{0}}{g_{e_{n}}} \bar{N}_{0^{m \lambda 2}}(h)+\underset{2_{e}}{h_{o_{n}}} \bar{M}_{e^{n \lambda 2}}(h)\right] \bar{N}_{e^{(1)}} e_{e^{n \lambda 2}}(-h)
\end{aligned}
$$

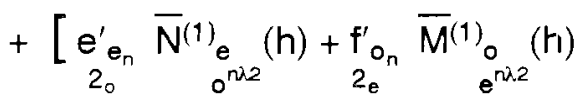

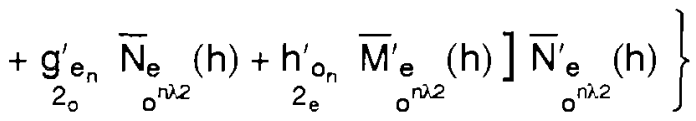

onde

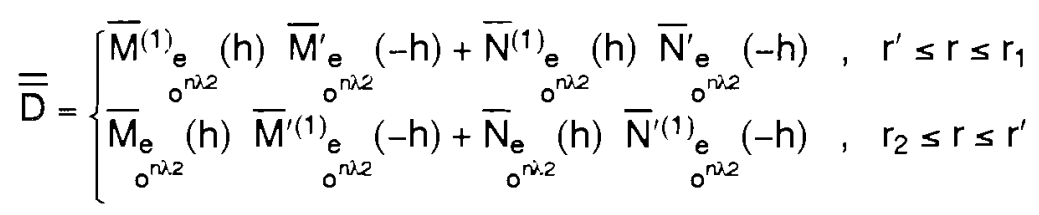

Regiāo 3:

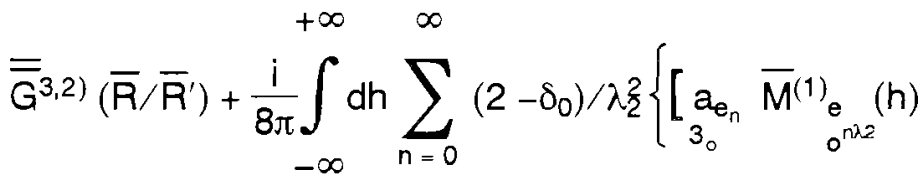

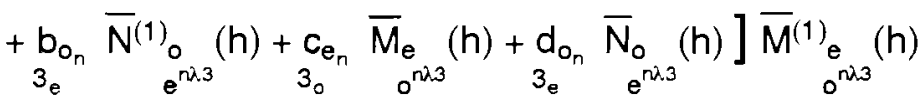

$$
\begin{aligned}
& +\underset{3_{0}}{\left[a_{e_{n}}\right.} \bar{M}_{0_{0}^{(1)}}^{\bar{M}_{e}}(h)+b_{e_{e}^{\prime}} o_{n} \bar{N}^{(1)} e_{e^{n \lambda 3}}(h)
\end{aligned}
$$

30 


$$
\begin{aligned}
& \left.+\underset{3_{0}}{c_{e_{n}}^{\prime}} \underset{o^{n \lambda 3}}{\bar{M}_{e}}(h)+\underset{3_{e}}{d_{o_{n}}^{\prime}} \bar{N}_{e^{n \lambda 3}}(h)\right] \bar{M}_{0^{n \lambda 3}}^{\prime}(-h)
\end{aligned}
$$

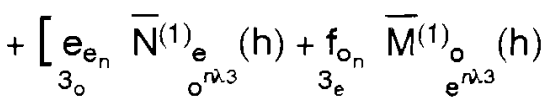

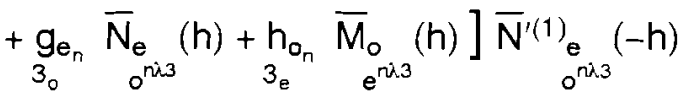

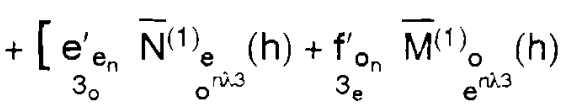

$$
\begin{aligned}
& \left.\left.+\underset{3_{0}}{g_{e_{n}}^{\prime}} \bar{N}_{o^{n \lambda 3}}(h)+h_{3_{e}^{\prime}}^{h_{o_{n}}} \bar{M}_{e^{n \lambda 3}}(h)\right] \underset{o^{n i 3}}{\bar{N}_{e}^{\prime}}(-h)\right\}
\end{aligned}
$$

Os índices superiores $(m, n)$, com $m=1,2,3$ e $n=2$, de $\overline{\bar{G}}^{(m, n)}\left(\bar{R} / \bar{R}^{\prime}\right)$ indicam as regiōes onde se localizam, respectivamente, os pontos de observaçāo e a fonte.

Os coeficientes de espalhamento, $a_{1}, b_{1}, \ldots, d_{2}, \ldots, g_{3}^{\prime}, h_{3}^{\prime}$, que aparecem em (7) até (9), são determinados através de sistemas de equaçóes, obtidos com a aplicaçāo das condiçōes de contorno nas interfaces, dadas por [1]:

para $r=r$

$$
\begin{aligned}
& \hat{r} \times \overline{\bar{G}}^{(1,2)}\left(\bar{R} / \bar{R}^{\prime}\right)=\hat{r} \times \overline{\bar{G}}^{(2,2)}\left(\bar{R} / \bar{R}^{\prime}\right) \\
& \frac{1}{\mu_{1}} \hat{r} \times \nabla \times \overline{\bar{G}}^{(1,2)}\left(\overline{\mathrm{R}} / \overline{\mathrm{R}}^{\prime}\right)=\frac{1}{\mu_{2}} \hat{\mathrm{r}} \times \nabla \times \overline{\overline{\mathrm{G}}}(2,2)\left(\overline{\mathrm{R}} / \overline{\mathrm{R}}^{\prime}\right)
\end{aligned}
$$

para $r=r_{2}$

$$
\begin{aligned}
& \hat{r} \times \overline{\overline{G^{(}}}(2,2)\left(\bar{R} / \overline{R^{\prime}}\right)=\hat{r} \times \overline{\bar{G}}(3,2)\left(\bar{R} / \bar{R}^{\prime}\right) \\
& \frac{1}{\mu_{2}} \hat{r} \times \nabla \times \overline{\bar{G}}(2,2)\left(\bar{R} / \bar{R}^{\prime}\right)=\frac{1}{\mu_{3}} \hat{r} \times \nabla \times \overline{\overline{G^{(}}}(3,2)\left(\bar{R} / \bar{R}^{\prime}\right)
\end{aligned}
$$


mant $=0$

$$
\because x(3,2)(9)=0
$$

As cypressces anaiticas destes coenicientes são fornecidas em [12].

2. Getcuso da Constimnte de rapropagação de uma Linha de Microfita nithation

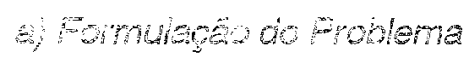

Sega una hing de microfita, de largura Wf, infinitamente longa na direção $z$, monade sobre substrato cilíndrico circular e localizada entre as regiōes $2 \mathrm{e}$ 3 , como instra a 5 . Para o caso de linhas finas, relativamente ao comprinenio de onda, pode-se assumir que apenas correntes elétricas na direçe 02 , heiepencontes de ф, fluem na linha. Esta aproximaçāo é válida para substros de undquer espessura [13]. Então, para uma linha simetricamente posionteda com relaca a $\$$, podemos considerar uma corrente superficial, Śadspor

$$
\vec{U}\left(\overrightarrow{\theta^{\prime}}\right)=\exp \left(k_{e} z^{\prime}\right) \delta\left(r^{\prime}-r_{2}\right) \cup\left(\mid \varphi^{\prime} l-\varphi_{1}\right) \hat{z}
$$

once $\varphi_{i}=W_{1} / 2 r_{2}$ e $k_{e}$ é a constante de propagaçāo da linha de microfita infinita, a ser ceiemineda. Aiunçāo $U\left(\left|\varphi^{\prime}\right|-\varphi_{f}\right)$ é a funçāo degrau unitário, deinide corro

$$
\mathcal{U}\left(\varphi^{\prime} \mid-\varphi_{i}\right)=\left\{\begin{array}{l}
1 \text { para }\left|\varphi^{\prime}\right| \leq \varphi_{i} \\
0 \text { para }|\varphi|>\varphi_{f}
\end{array}\right.
$$

O campo elemiso na regiäo 2 , para uma fonte de corrente elétrica qualquer, édedopoing

$$
E_{2}(\bar{\beta})=\varepsilon v_{2} \iiint^{\prime} \bar{G}(2,2)\left(\bar{R} / \overline{R^{\prime}}\right) \cdot \bar{J}\left(\overline{R^{\prime}}\right) d v^{\prime}
$$




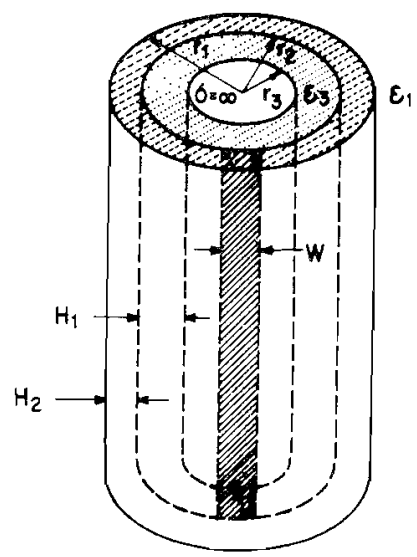

Figura 2. Linha de microfita sobre substrato cilíndrico circular, com cobertura dielétrica.

Uma vez que as componentes tangenciais do campo elétrico são desprezíveis na superfície de um bom condưtor, a substituiçāo de (15) em (17) permite que se escreva para a componente longitudinal

$$
\begin{gathered}
\iint_{s^{\prime}} \frac{i}{8 \pi} \int_{-\infty}^{\infty} d h \sum_{n=0}^{\infty}\left(2-\delta_{0}\right) / \lambda_{2}^{2}\left[\left(e_{2 e n} N_{z e n}^{(1)}+\left(1+g_{2 e n}\right) N_{z e n}\right) N_{z e n}^{\prime 1}\right. \\
+\left(e_{2 o n} N_{z o n}^{(1)}+\left(1+g_{2 o n}\right) N_{z o n}\right) N_{z o n}^{\prime 1)}+\left(e_{2 \text { en }}^{\prime} N_{z e n}^{(1)}+g_{\text {zen }}^{\prime} N_{z e n}\right) N_{z e n}^{\prime} \\
\left.+\left(e_{2 o n}^{\prime} N_{z o n}^{(1)}+g_{\text {zon }}^{\prime} N_{z o n}\right) N_{z o n}^{\prime}\right] \exp \left(i k_{e} z^{\prime}\right) r_{2} d \varphi^{\prime} d z^{\prime}=0 \\
\text { para } r=r^{\prime}=r_{2}
\end{gathered}
$$

onde as funçōes $\bar{M}$ e $\bar{N}$, dadas por (5) e (6), são escritas nas seguintes formas simplificadas

$$
\begin{aligned}
& \bar{M}_{\text {on } \lambda_{2}}(h)=M_{\text {ron }} \hat{r}+M_{\text {qon }} \hat{\varphi} \\
& \bar{M}_{\text {en } \lambda_{2}}(h)=M_{\text {ren }} \hat{r}+M_{\text {qen }} \hat{\varphi} \\
& \bar{N}_{\text {on } \lambda_{2}}(h)=N_{\text {ron }} \hat{r}+N_{\text {qon }} \hat{\varphi}+N_{\text {zon }} \hat{z}
\end{aligned}
$$




$$
\bar{N}_{\text {enzz }}(h)=N_{\text {ren }} \hat{r}+N_{\text {qen }} \hat{\varphi}+N_{z e n} \hat{z}
$$

A integral em $h$ ern (18), é discretizada para $h=k_{\varepsilon}$ [14]. Anulando-se a integral em $\phi$ em (18), automaticamente torna-se nulo o integrando para $z$ '. Resolvendo-se a integral em ' $\phi^{\prime}$, a constante de propagaçāo é obtida, procurando-se o valor de $k_{e}$, que anula a integral da componente longitudinal ao longo da seçāo transversal da linha, ou seja, resolvendo-se para $k_{e}$ a equaçāo

$$
\begin{aligned}
& \sum_{n=0}^{\infty}\left(2-\delta_{0}\right) \Psi z J_{n}\left(\lambda_{2} r_{2}\right)\left(1+g_{2 e}+e_{2 e}^{\prime}\right. \\
& \left.+e_{2 e} H_{n}^{(1)}\left(\lambda_{2} r_{2}\right) / J_{n}\left(\lambda_{2} r_{2}\right)+g_{2 e}^{\prime} J_{n}\left(\lambda_{2} r_{2}\right) / H_{n}^{(1)}\left(\lambda_{2} r_{2}\right)\right)=0
\end{aligned}
$$

onde

$$
\Psi_{f}=\int_{-\varphi_{f}}^{\varphi_{f}} \cos (n \varphi) d \varphi
$$

Uma vez conhecida a constante de propagação da linha de microfita, a constante dielétrica efetiva é obtida da relaçāo

$$
\varepsilon_{\mathrm{ef}}=\left(k_{e} / k_{0}\right)^{1 / 2}
$$

onde ko é a constante de propagaçáo do espaço livre.

\section{b) Resultados Numéricos}

A dependência da constante dielétrica efetiva em funçāo de $k_{0} r_{2}$ para $\varepsilon_{1}=\varepsilon_{0}, \varepsilon_{2}=\varepsilon_{3}=9,6 \varepsilon_{0}$ ( $\varepsilon_{0}$ é a permissividade do espaço livre) e para várias alturas de cobertura dielétrica, é mostrada na Fig. 3. Na Fig. 4, a constante dielétrica efetiva é mostrada em funçāo de $\mathrm{H}_{2} \mathcal{W}_{\mathrm{f}}$, para vários valores de permissividade da cobertura dielétrica, $\varepsilon_{2}$, e para $\varepsilon_{1}=\varepsilon_{0}, \varepsilon_{3}=9,6 \varepsilon_{0}$ e $k_{0} r_{2}=1$. 


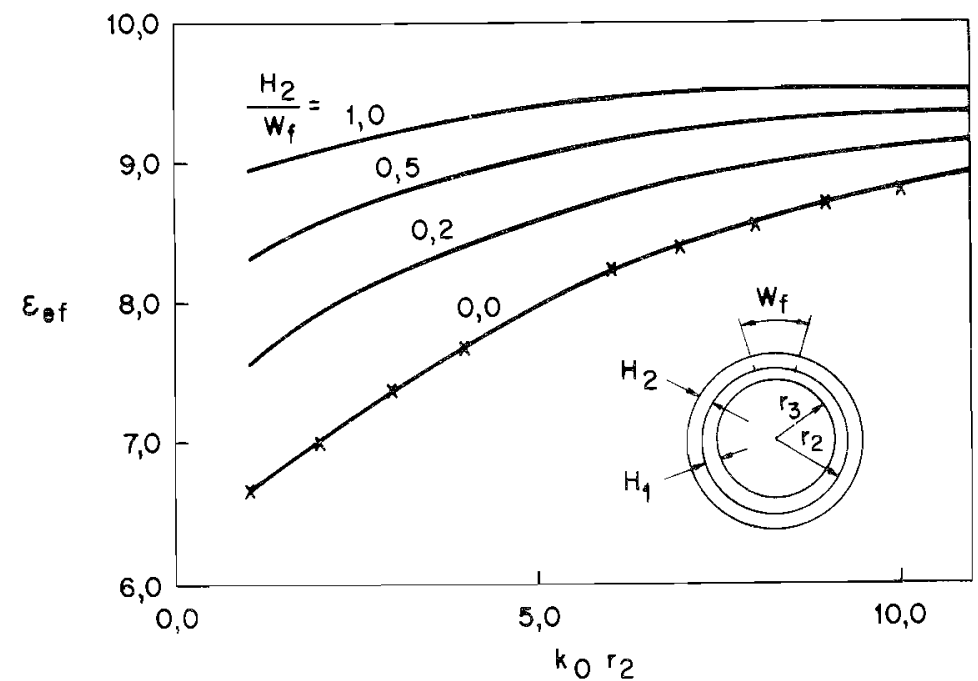

Figura 3. Constante dielétrica efetiva, eef, em funçāo de $k_{0}$ r2, para vários valores de $H_{2} / W_{f}$ e para $\varepsilon_{1}=\varepsilon_{0}, \varepsilon_{2}=\varepsilon_{3}=9,6 \varepsilon_{0}, H_{1}=W_{f}$, e $r_{3} / r_{2}=0,9$.

Resultados de [4] são mostrados com marcas $x$.

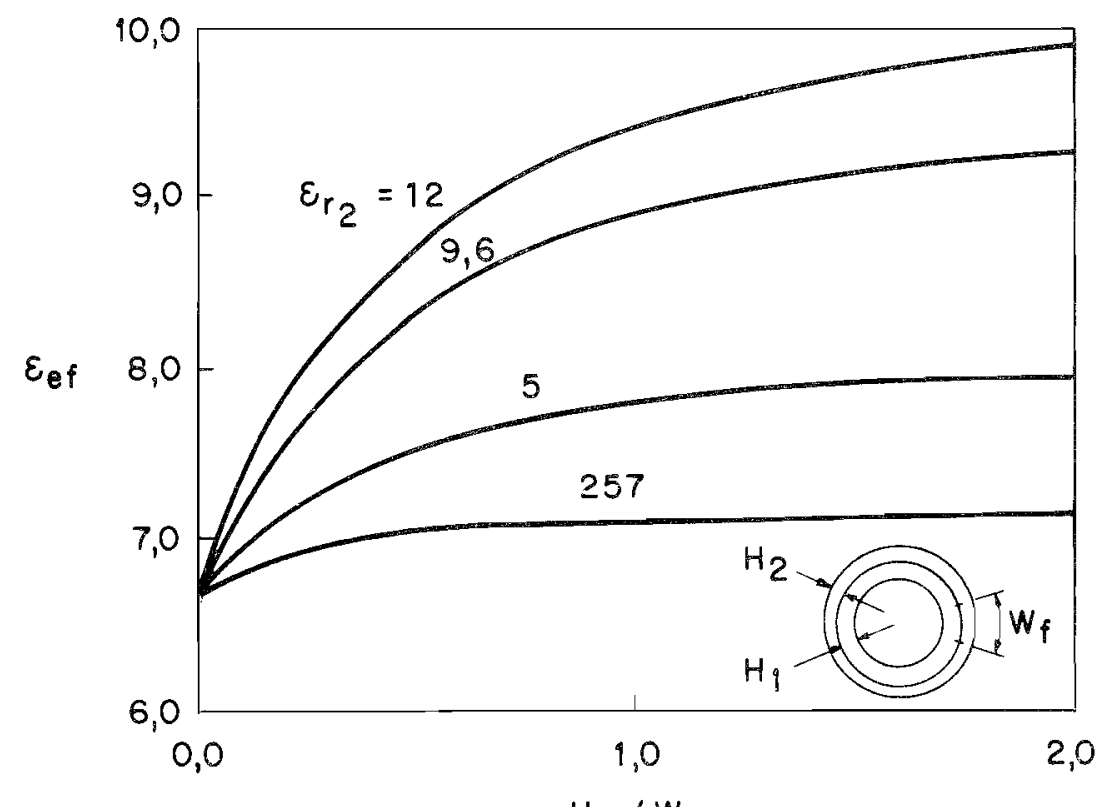

$\mathrm{H}_{2} / \mathrm{W}_{\mathrm{f}}$

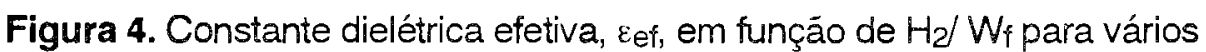
valores de $\varepsilon_{2}$ e para $\varepsilon_{1}=\varepsilon_{0}, \varepsilon_{3}=9,6 \varepsilon_{0}, H_{1}=W_{f}, k_{0} r_{2}=1$ e $r_{3} / r_{2}=0,9$. 
Na Fig. 3, os resultados de Nakatanie Alexopoulos [4] sāo também mostrados e a concordância é observada quando a altura da cobertura dielétrica é reduzida a zero $\left(\mathrm{H}_{2}=0\right)$. Nota-se que com a escolha de $\varepsilon_{2}=9,6 \varepsilon_{0}$, o efeito da cobertura dielétrica sobre a constante dielétrica efetiva é apreciável mesmo para pequenos valores de $k_{0} r_{2}$. Nota-se também na Fig. 4, que para $k_{0} r_{2}=1$, as curvas de $\varepsilon_{\text {ef }}$ aproximam-se de valores assintóticos para valores de $\mathrm{H}_{2}$ acima de 1,9 $\mathrm{W}_{\uparrow}$ para todos os valores de $\varepsilon_{2}$ usados.

Sendo $r_{3}=2,5 \mathrm{~cm}, W_{f}=0,477 \mathrm{~cm}$, a Tabela 1 fornece $o$ valor de h no eixo real que estabelece a localizaçáo do pólo da onda de superfície, a constante de propagação do espaço livre, $k_{0}$, a constante de propagação da regiāo 3 , $k_{3}$, a constante de propagaçāo da linha de microfita infinita, $k_{e}$, para $H_{1}=0,159$ $\mathrm{cm}, \varepsilon_{1}=\varepsilon_{2}=\varepsilon_{0}, \varepsilon_{3}=2,57 \varepsilon_{0}$, nas freqüências de $2,20,2,25,2,30$ e $2,35 \mathrm{GHz}$

\begin{tabular}{lcccc}
\hline Freqüência $(\mathrm{GHz})$ & Pólo $(\mathrm{h})\left(\mathrm{m}^{-1}\right)$ & $k_{0}\left(\mathrm{~m}^{-1}\right)$ & $k_{3}\left(\mathrm{~m}^{-1}\right)$ & $k_{\mathrm{e}}\left(\mathrm{m}^{-1}\right)$ \\
\hline 2,20 & 46,507 & 46,08 & 73,87 & 67,8 \\
2,25 & 47,569 & 47,12 & 75,54 & 69,3 \\
2,30 & 48,632 & 48,17 & 77,22 & 70,9 \\
2,35 & 49,696 & 49,22 & 78,91 & 72,4 \\
\hline
\end{tabular}

Tabela 1. Valores de h que caracterizam a localizaçāo do pólo de onda de superfície e da constante de propagaçāo de uma linha de microfita, $\mathrm{k}_{\boldsymbol{\varepsilon}}$, com $W_{f}=0,477 \mathrm{~cm}$ sobre substrato cilíndrico circular com $\mathrm{r}_{3}=2,5 \mathrm{~cm}$ e $H_{1}=0,159 \mathrm{~cm}$, sendo $\varepsilon_{1}=\varepsilon_{2}=\varepsilon_{0}$ e $\varepsilon_{3}=2,57 \varepsilon_{0}$.

Na Tabela 2 têm-se resultados para as mesmas grandezas da Tabela 1 , porém com cobertura dielétrica, fazendo-se $H_{1}=H_{2}=0,159 \mathrm{~cm}, \varepsilon_{1}=\varepsilon_{0}$ e $\varepsilon_{2}=\varepsilon_{3}=2,57 \varepsilon_{0}$. A espessura do substrato foi selecionada de forma que apenas o primeiro modo da onda de superfície seja excitado.

Dos resultados das tabelas 1 e 2 nota-se a influência da cobertura dielétrica na constante de propagaçāo da linha e, neste caso, sendo ke maior que o valor de h que caracteriza a localização do pólo, nenhuma potência de onda de superfície é gerada. Considera-se a linha de microfita uniforme, pois descontinuidades na linha podem excitar modos da onda de superfície, bem como modos propagantes além do fundamental [13]. 


\begin{tabular}{|c|c|c|c|c|}
\hline Freqüência & Póto $(h)\left(m^{-1}\right)$ & $k o\left(m^{-1}\right)$ & 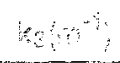 & Kon \\
\hline 2,20 & 47,129 & 46,08 & $730 \%$ & 74 \\
\hline 2,25 & 48,216 & $47: 12$ & 35.5 & 83 \\
\hline 2,30 & 49,305 & 48,17 & 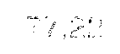 & $\because$, \\
\hline 2,35 & 50,396 & 4022 & 7068 & 8 \\
\hline
\end{tabular}

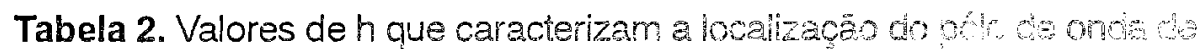

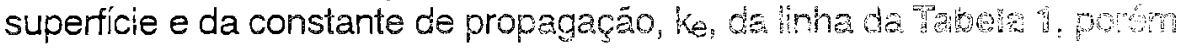
$\mathrm{com} \mathrm{H}_{1}=\mathrm{H}_{2}=0,159 \mathrm{~cm}, \varepsilon_{1}=\varepsilon_{0}$ e $\varepsilon_{2}=\varepsilon_{3}=2,0,00$.

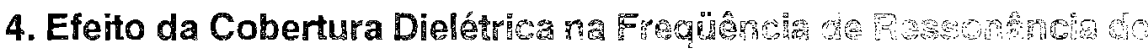 uma Antena de Microfita de Forma Rerameng:}

\section{a) Determinaçāo da Corrente de superfís}

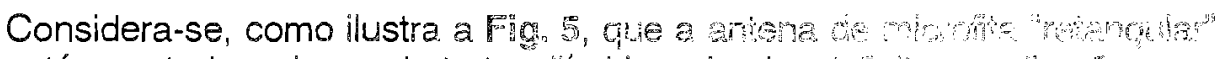
está montada sobre substrato elindrico dicular thrm $n$

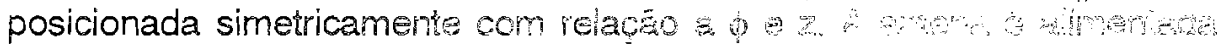

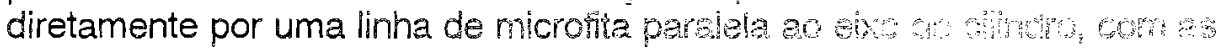

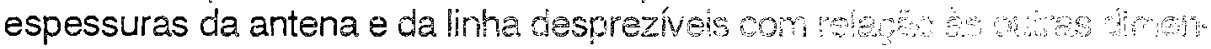

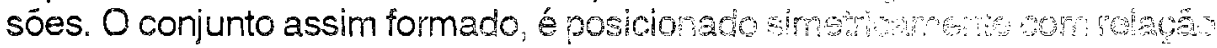
a $\phi$, na interface entre as regioes 2 e 3 da

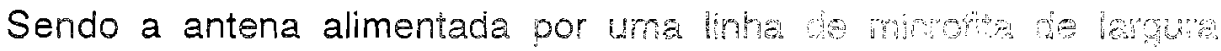

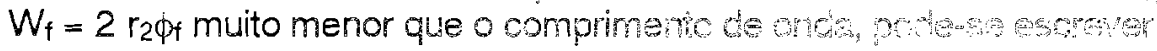

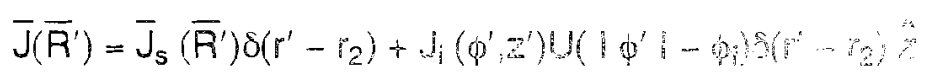

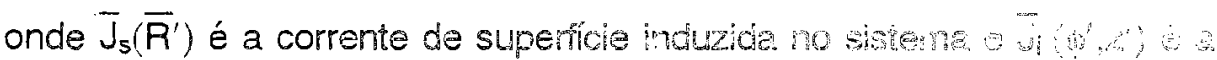

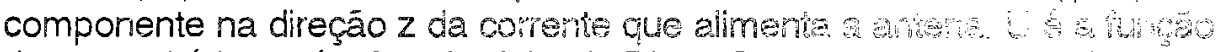

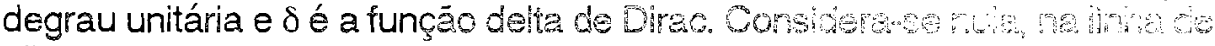
alimentaçáo, a componente na direçāo $\phi$.

Substituindo (26) em (17) e aplicando-se a condiçảr de contomo de carmo elétrico nulo sobre a antena e a linha de alimentacáo, consideradas concín toras perfeitas, obtém-se a seguinte equação integra para ds (a') 


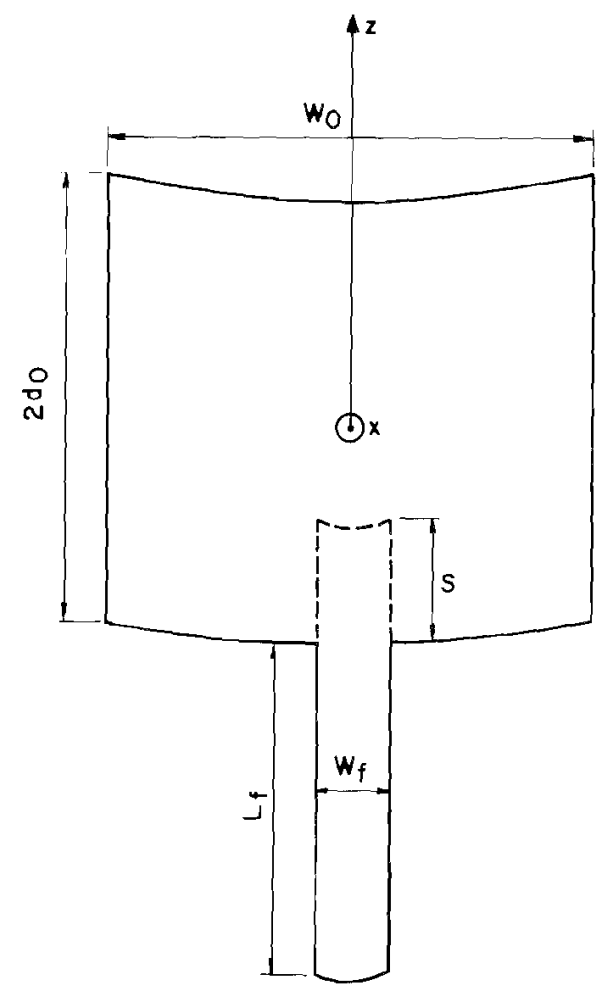

Figura 5. Antena de microfita "retangular" alimentada por uma linha de microfita.

$$
\begin{aligned}
& {\left[\iint_{s^{\prime}} \overline{\bar{G}}(2,2)\left(\bar{R} / \overline{R^{\prime}}\right) \cdot \bar{J}_{s}\left(\overline{R^{\prime}}\right) d s^{\prime}\right]_{\tan }} \\
& =-\left[\iint_{s^{\prime}} \overline{\bar{G}}(2,2)\left(\bar{R} / \overline{R^{\prime}}\right) \cdot J_{i}\left(\phi^{\prime}, z^{\prime}\right) \hat{z} d s^{\prime}\right]_{\tan }, \quad p a r a \begin{array}{r}
r=r^{\prime}=r_{2} \\
\left|\phi^{\prime}\right|<\phi_{f}
\end{array}
\end{aligned}
$$

onde $d s^{\prime}=r_{2} d \phi^{\prime} d z^{\prime}$

Em (27), o primeiro membro é o campo elétrico tangencial espalhado e o segundo membro é o campo elétrico tangencial incidente. 
A solução da equação em (27), pelo método dos momentos, consiste em expandir a corrente de superfície, $J_{s}\left(R^{\prime}\right)$, em séries de funçōes, discretizando a seguir a equaçāo resultante com o uso de funçōes de teste, que podem ser iguais as de expansāo (método de Galerkin), obtendo-se desta forma uma equaçāo matricial do tipo

$$
[\mathrm{Z}][\mathrm{I}]=[\mathrm{V}]
$$

onde $[Z]$, [] e [V] são, respectivamente, as matrizes impedância, de corrente e de voltagem generalizada do método dos momentos [15].

As correntes na linha de alimentação e na antena são expandidas em três tipos de modos [8]: modos de ondas caminhantes sobre a linha de alimentação, modos senoidais por partes na regiāo da junçāo e modos de domínio completo sobre a antena.

Para o caso de linha de alimentaçāo fina, relativamente ao comprimento de onda guiado, pode-se assumir que apenas correntes elétricas na direçáo $z$ fluem na linha de microfita [13]. Entāo, define-se a corrente elétrica incidente como sendo

$$
\overline{J i n c}_{(\phi, z)}=\frac{1}{W_{f}} \exp \left(i k_{e}\left(z+d_{0}\right)\right) \hat{z}
$$

e a corrente refletida fica

$$
\bar{J}^{\text {ref }}(\phi, z)=-\frac{R}{W_{f}} \exp \left(-i k_{e}\left(z+d_{0}\right)\right) \hat{z}
$$

onde $R$ é o coeficiente de reflexāo referenciado no final da linha, na junçāo entre a linha de microfita e a antena, a ser determinado; $W_{1}$ é a largura da linha de alimentaçāo; do é o meio comprimento da antena na direçāo z; e ke é a constante de propagaçāo da linha de microfita infinita. Os comprimentos dos modos de onda caminhante, $L_{f}$, săo feitos múltiplos de $\lambda_{e} / 2$, onde $\lambda_{e} e$ é o comprimento de onda guiado na linha, sendo estes modos separados em termos de senos e cossenos, e, truncados de forma que nenhuma descontinuidade exista no final do modo [13]. 
Na regiāo próxima à junçāo, onde a linha de alimentaçāo se une à antena, a corrente não é uniforme por causa da descontinuidade. Funçōes senoidais por partes, sobrepondo a linha de alimentaçāo e a antena, sāo usadas para modelar esta nāo uniformidade. Entāo, define-se

$$
\text { Jjun }(\phi, z)=\sum_{m=1}^{M} I_{m} J_{j}(\phi, z) \hat{z}
$$

sendo

$$
J_{j}(\phi, z)=\frac{1}{W_{f}} \frac{\operatorname{sen} k\left(I-\left|z-z_{n}\right|\right)}{\operatorname{sen} k l} \text {, para }\left|z-z_{n}\right|<l,|\phi|<\phi_{f}(32)
$$

onde $z_{n}$ é a coordenada do centro do modo e / é igual à metade do comprimento do modo. A constante $k$ é arbitrária, e, por conveniência foi escolhida igual a $\mathrm{k}_{\mathrm{e}}$.

Expandindo-se a corrente sobre a antena em termos de uma série de funçỏes senoidais de domínio completo, tem-se

$$
\bar{J}^{\text {ant }}(\phi, z)=\sum_{p=1}^{P} K_{p} P_{z}(\phi, z) \hat{z}+\sum_{q=1}^{Q} L_{q} P_{\phi}(\phi, z) \hat{\phi}
$$

sendo

$$
\begin{gathered}
\left.P_{z}(\phi, z)=\frac{1}{W_{0}} \operatorname{sen}\left(p \pi\left(z+d_{0}\right) / 2 d_{0}\right)\right) \text { para }|z|<d_{0},|\phi|<\phi_{0}(34) \\
p=1,2, \ldots, P
\end{gathered}
$$

e

$$
\begin{gathered}
\left.P_{\phi}(\phi, z)=\frac{1}{2 d_{0}} \operatorname{sen}\left(q \pi\left(z+\phi_{0}\right) / 2 \phi_{0}\right)\right) \text { para }|z|<d_{0},|\phi|<\phi_{0} \\
q=1,2, \ldots, Q
\end{gathered}
$$


onde $\phi_{0}=W_{0} / 2 r_{2}$, sendo $W_{0} \circ$ comprimento da antena na direção $\phi$.

Observa-se que estes modos de expansão nāo contêm qualquer variaçāo na direçāo ortogonal à direçāo do fluxo de corrente, e a expressāo para levar em conta o efeito de borda nāo foi incluída, pois, embora melhore a convergência do método dos momentos, há um aumento de complexidade na integração [8].

O uso de (29) até (31) e (33) em (27), com a aplicaçāo do método dos momentos, resulta na equaçāo matricial em (28), repetida aqui de forma mais explicativa

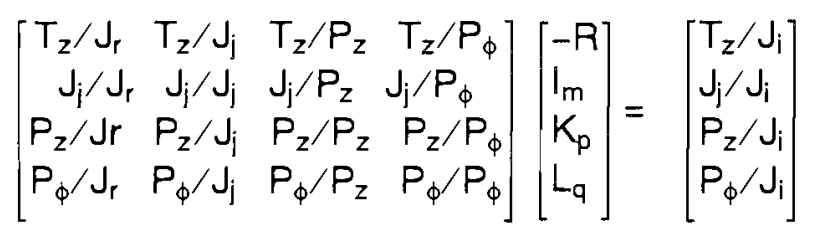

onde:

$T_{z}$ é uma funçāo de teste sobre a linha de alimentaçāo (uma única funçāo senoidal por partes);

$J_{j}$ é uma série de $M$ funçōes senoidais por partes na junção;

$P_{z}$ é uma série de $P$ funçōes de domínio completo para as correntes na direçāo $z$ da antena;

$P_{\phi}$ é uma série de $Q$ funçōes de domínio completo para correntes na direçăo ф na antena;

$J_{i}$ é o modo de onda de corrente caminhante incidente;

$J_{\mathrm{r}}$ é o modo de onda de corrente caminhante refletida;

-R é a amplitude da onda de corrente refletida na linha, a ser determinada;

$I_{m}, K_{p}$ e $L_{q}$ sáo os coeficientes de expansāo a serem determinados.

A incógnita $-\mathrm{R}$ faz com que o número de incógnitas seja maior que o número de equaçōes. Entāo, para se obter a igualdade, acrescenta-se a funçāo de teste $T_{z}$ [8]. Nota-se que para o cálculo da matriz impedância generalizada 
em (36), existem quatro combinaçōes diferentes entre as funçōes de teste e o modo de onda caminhante refletida, e doze combinaçōes distintas entre as funçōes de teste e as funçōes de expansāo. Para a determinaçāo da matriz voltagem generalizada, existem quatro combinaçōes entre as funçōes de teste e o modo de onda caminhante incidente. Os coeficientes de expansáo da corrente e o coeficiente de reflexão são obtidos da equação

$$
[1]=[Z]^{-1}[\mathrm{~V}]
$$

Uma vez conhecido o coeficiente de reflexão de voltagem, $R$, a resistência $r$ e a reatância $x$ de entrada da antena, normalizadas com relaçáo à impedância característica da linha de alimentação, sāo dadas por [16]

$$
r+i x=(1+R) /(1-R)
$$

\section{b) Resultados Numéricos}

As respostas obtidas através da aplicaçáo do método dos momentos, que soluciona a equação em (27), sáo dependentes do comprimento finito da linha de microfita, Lt, das convergências das integrais em he dos somatórios das funçōes de Bessel, de Hankel e das funçōes circulares cos $(n \phi)$ e sen $(n \phi)$, da distância $S$ em que as funçōes senoidais por partes se sobrepóem na antena, do meio comprimento / destas funçōes, e da quantidade de termos usados nas funçōes de expansáo.

As integrais em $\mathrm{h}$ sāo integrais no eixo real, onde atenção especial tem que ser dada, buscando a contribuiçāo dos pólos de onda de superfície. A complexidade das expressōes tornou inviável o uso de métodos analíticos [17] e [18] no cálculo da contribuição do pólo de onda de superfície. Para se obter a contribuiçāo do integrando na regiāo do pólo, consideram-se pequenas perdas no dielétrico, fazendo-se $\varepsilon^{\prime}{ }_{3}=\varepsilon_{3}(1+i \mathrm{tg} \delta)$ [16], resultando que Im $\left(k_{3}\right)>0$. Este procedimento desloca o pólo do eixo real [1], facilitando a integraçāo numérica. Um valor de tg $\delta=0,001$ foi usado, uma vez que testes de convergência mostraram que valores menores aumentam o tempo de processamento sem que se alterem os resultados. $O$ intervalo de integração nāo é crítico, podendo-se integrar de $k_{1}$ a $2 \zeta_{p}-k_{1}$ [19], onde $\zeta_{p}$ é o valor de h na localizaçāo do pólo.

As integrais em $h$ das funçōes de Green apresentaram convergência dos resultados computando as contribuiçóes dos integrandos de $h=-80 k_{0} a$ 
$h \approx 80 k_{0}$ ( $k_{0}$ é a constante de propagação do espaço livre). A consideração do somatório dos termos das funçōes acima de n igual a dezesseis teve efeito desprezível nos resultados.

Pozar e Voda [8] constataram no estudo da antena de microfita retangular, alimentada por uma linha de microfita, sobre substrato plano, que as respostas obtidas analiticamente nāo sāo extremamente sensíveis a valores de $S$ e do tais que $0,5 \leq S / 2 d_{0} \leq 0,65$, verificando-se que dentro dessa faixa há uma boa concordância com os resultados experimentais. A Fig. 6 mostra a

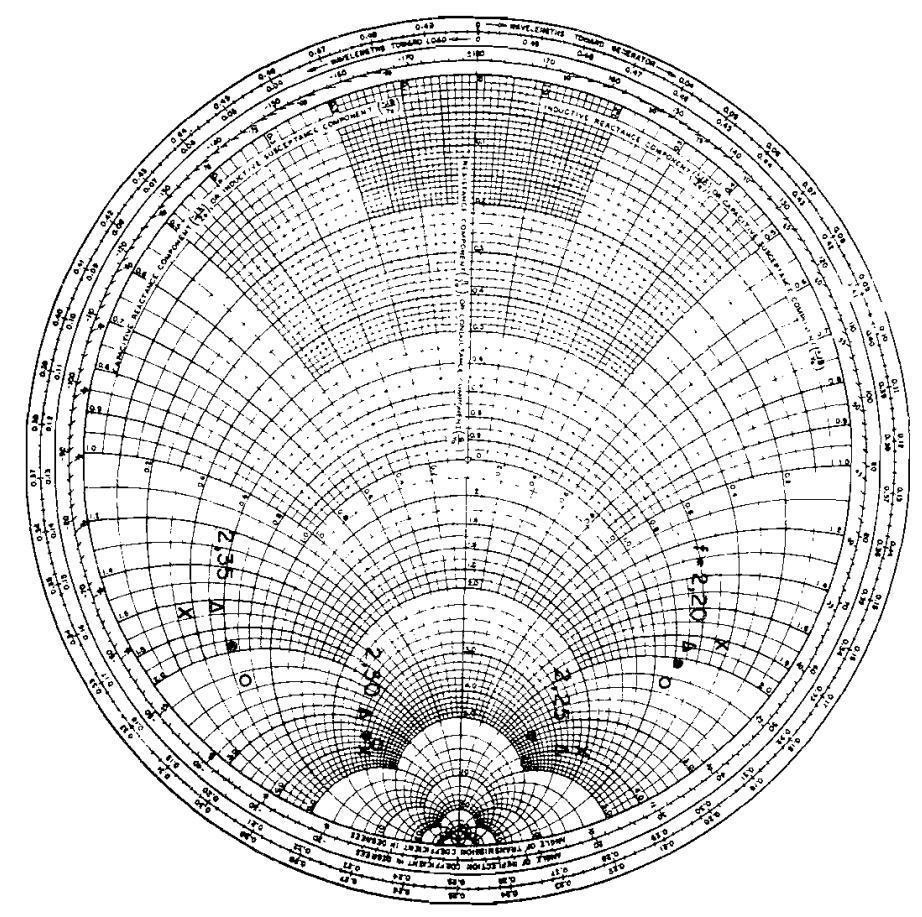

Figura 6. Impedância de entrada de uma antena de microfita "retangular" com $2 \mathrm{~d}_{0}=W_{0}=4,02 \mathrm{~cm}$, alimentada diretamente por uma linha de microfita de largura $W_{f}=0,477 \mathrm{~cm}, c o m r_{3}=2,5 \mathrm{~cm}, r_{2}=2,659 \mathrm{~cm}$, $\varepsilon_{1}=\varepsilon_{2}=\varepsilon_{0}, \varepsilon_{3}=2,57 \varepsilon_{0}$, nas freqüências de 2,$20 ; 2,25 ; 2,30$ e $2,35 \mathrm{GHz}$, sendo:

$\triangle$ calculado para $S / 2 d_{0}=0,45$;

- calculado para $S / 2 d_{0}=0,55$;

- calculado para $S / 2 d_{0}=0,65$;

$x$ valores medidos [8] para antena com as mesmas dimensōes, porém sobre substrato plano. 
impedância de entrada para uma antena de forma "retangular", com $2 \mathrm{~d}_{0}=W_{0}=4,02 \mathrm{~cm}$, alimentada diretamente por uma linha de microfita com $W_{f}=0,477 \mathrm{~cm}$, posicionada na interface das regiöes 2 e $3 \mathrm{da}$ Fig. 1, sendo $r_{3}=2,5 \mathrm{~cm}, r_{2}=2,659 \mathrm{~cm}, \varepsilon_{3}=2,57 \varepsilon_{0}$ ( $\varepsilon_{0}$ é a permissividade do espaço livre), $\varepsilon_{2}=\varepsilon_{1}=\varepsilon_{0}$, calculada nas freqüências de 2,$20 ; 2,25 ; 2,30$ e $2,35 \mathrm{GHz}$, para valores de $S / 2 d_{0}$ iguais a 0,45; 0,55 e 0,65. Resultados medidos por Pozar e Voda [8], para a antena sobre substrato plano, também sáo mostrados. A concordância dos resultados mostra que a freqüência de ressonância da antena nāo é fortemente afetada pela curvatura do substrato.

As Figuras 7 e 8 apresentam, respectivamente, a resistência e reatância de entrada da antena descrita anteriormente, normalizadas com relação à impedância característica da linha de alimentaçāo, em funçāo da freqüểncia. São considerados os casos sem cobertura, com cobertura dielétrica de espessura $H / 2$ e $H$, onde $H=r_{2}-r_{3}$, sendo a permissividade elétrica da cobertura, $\varepsilon_{2}=2,57 \varepsilon_{0}$. Nota-se 0 efeito da cobertura dielétrica na freqüência de ressonância da antena, alterando sensivelmente esta freqüência, mesmo para uma espessura de $\mathrm{H} / 2$.

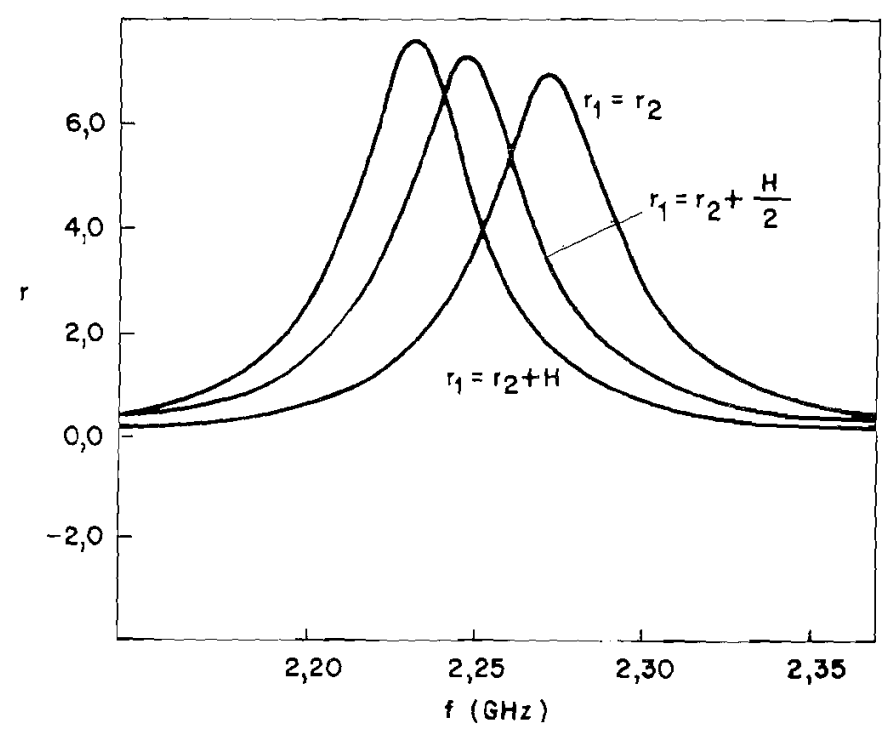

Figura 7. Resistência de entrada, normalizada com relação à impedância característica da linha de alimentaçāo, em funçāo da freqüência, para a antena da Fig. 3, para valores de cobertura dielétrica de $0, H / 2$ e $H$, sendo $H=0,159 \mathrm{~cm}$ e $\varepsilon_{2}=2,57 \varepsilon_{0}$.

Os cálculos foram feitos com uma relaçāo de $S / 2 d_{0}=0,55$. 


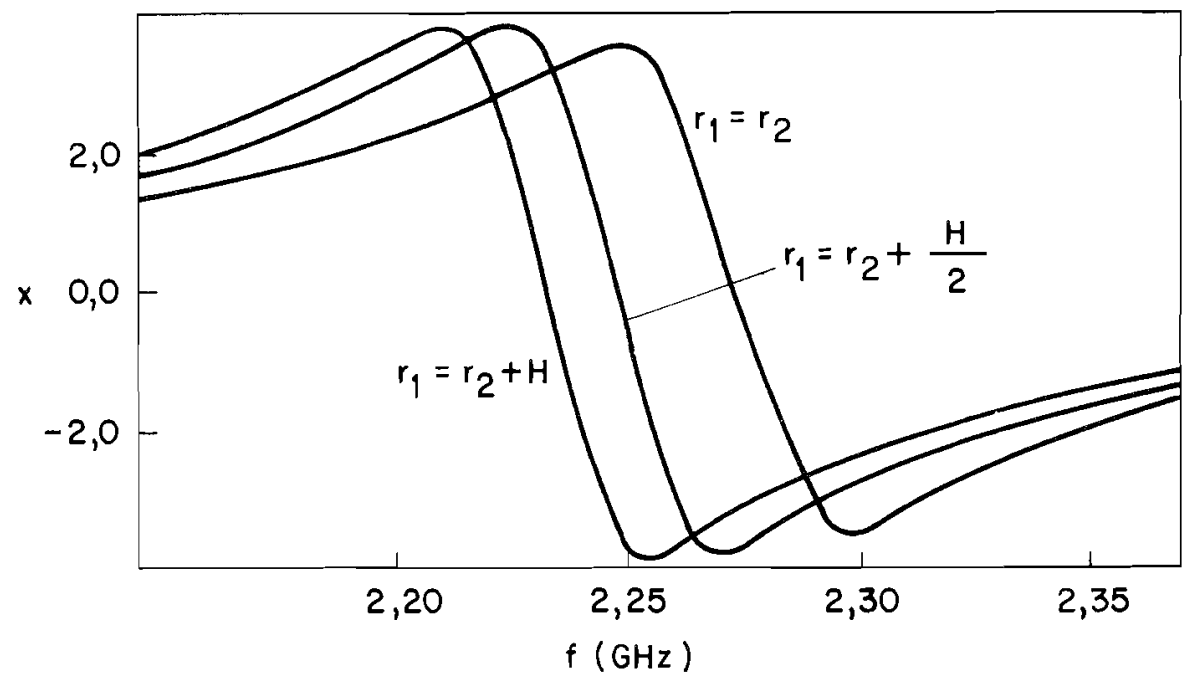

Figura 8. Reatância de entrada, normalizada com relaçāo à impedância característica da linha de alimentaçāo, em funçāo da freqüência, para a antena da Fig. 3.

Os cálculos foram feitos com uma relaçāo de $S / 2 d_{0}=0,55$.

As respostas obtidas apresentam convergências para comprimentos finitos do modo de onda caminhante, $L_{f}$ (comprimento finito da linha de microfita) acima de $3 \lambda_{e}$. Convergiram também com o uso de oito funçōes senoidais por partes na regiāo próxima à junçāo, de meio comprimento $I=0,05 \lambda_{e}$, três modos pares de domínio completo na direçāo $z$ e três modos ímpares na direçáo $\phi$. Os números de convergências dos resultados obtidos concordam com os fornecidos por Pozar e Voda [8].

As constantes de propagação da linha de alimentação e as localizaçōes dos pólos da onda de superfície, para as freqüências de 2,20;2,25;2,30 e 2,35 $\mathrm{GHz}$, para as análises da antena sem cobertura e com cobertura de espessura $H=0,159 \mathrm{~cm}$, sāo dados, respectivamente, nas tabelas 1 e 2 .

\section{Conclusões}

O formalismo das funçōes de Green diádicas foi utilizado neste trabalho, para se analisar o comportamento dos campos eletromagnéticos em meios divi- 
didos em quatro camadas cilíndricas concêntricas, sendo a camada central um condutor perfeito.

Como aplicaçōes da formulaçáo geral, dois casos particulares foram analisados: a linha de microfita infinita sobre substrato cilíndrico circular e a antena de microfita de forma "retangular", alimentada diretamente por uma linha de microfita, também sobre substrato cilíndrico circular. Em ambos os casos, foi examinado o efeito da cobertura dielétrica, seja na constante de propagaçáo do modo fundamental da linha, seja na freqüência de ressonância da antena.

Os resultados apresentados neste trabalho mostram que a cobertura dielétrica exerce uma forte influência nas carcterísticas elétricas dos dispositivos analisados. Notam-se modificaçōes substanciais na freqüência de ressonância da antena e na constante de propagaçāo da linha, mesmo considerando uma cobertura com baixo valor de permissividade e espessura muito menor que o comprimento de onda.

É importante observar a nāo existência de dados experimentais disponiveis, a respeito do efeito da cobertura dielétrica nas características elétricas das antenas de microfita, sobre substratos cilíndricos circulares, para comparação com os resultados deste trabalho. Porém, o uso das potencialidades das funçōes de Green diádicas e do método dos momentos, nas soluçōes de problemas eletromagnéticos e o confronto dos resultados numéricos obtidos para a linha de microfita com os de Nakatani e Alexopoulos [4], que mostram uma perfeita concordância quando a espessura da cobertura é reduzida a zero, tornam confiáveis os resultados apresentados.

As análises foram feitas para dielétricos supostos não magnéticos, tais que $\mu_{1}=\mu_{2}=\mu_{3}=\mu_{0}$, onde $\mu_{0}$ é a permeabilidade do espaço livre.

\section{Referências}

[1] C.T. Tai, "Dyadic Green's Functions in Electromagnetic Theory", Intext Publishers, Scranton, Pensylvannia, 1971.

[2] C.T. Tai, "Eigen-Function Expansion of Dyadic Green's Functions", Math. Note 28, The University of Michigan Radiation Laboratory, Ann Harbor, Michigan, 1973. 
[3] C.T. Tai, "The Singular Terms in the Eigen-Function Expansion of Dyadic Green's Functions of the Electric Type", The University of Michigan Radiation Laboratory, Ann Arbor, Michigan, 1980.

[4] A. Nakatani e N.G. Alexopoulos, "Microstrip Circuit Elements on Cylindrical Substrates", IEEE-MTT-S Microwave Theory and Techniques International Symposium Digest, Las Vegas, Estados Unidos, 1987, pp. 739-742.

[5] R.F. Harrington. "Field Computation by Moment Methods", The Macmillan Company. New York, 1968.

[6] M.D. Deshpande e M.C. Bailey, "Input Impedance of Microstrip Antennas", IEEE Transactions on Antennas and Propagation, vol. AP. 30,1982 , pp. 645-650.

[7] D.M. Pozar, "Input Impedance and Mutual Coupling of Rectangular Microstrip Anternas", IEEE Transactions on Antennas and Propagation, vol. AP-30, 1982. pp. 1191-1196.

[8] D.M. Pozar e S.M. Voda, "A Rigorous Analysis of a Microstripline Fed Patch Antenna", IEEE Transactions on Antennas and Propagation, vol. AP-35, 1987, pp. 1343-1350.

[9] A.J.M. Soares, "Análise da Propagaçāo de Ondas em Meios com Multicamadas Cilíndricas Concêntricas Ultilizando-se Funçōes de Green Diádicas", Tese de Doutorado, Departamento de Engenharia Elétrica, Universidade Estadual de Campinas, Campinas, SP, 1986.

[10] A.H. Mohammadian, "Integral Equation Formulation for Printed Antennas", IEEE Antennas and Propagation Symposium Digest, Estados Unidos, 1987, pp. 606-609.

[11] S.B.A. Fonseca e A.J. Giarola, "Dyadic Green's Functions and Their Use in the Analysis of Microstrip Antennas", em Advances in Electronics and Electron Physics, editado por Peter W. Hawkes, vol. 65, Academic Press, 1985, pp. 1-90.

[12] F.C. Silva, "Aplicação do Formalismo das Funçōes de Green Diádicas e do Método dos Momentos, no Estudo dos Elementos de Microfita sobre Substratos Cilíndricos Circulares", Tese de Doutorado, Departamento de Microonda e Óptica, Faculdade de Engenharia Elétrica, Universidade Estadual de Campinas, Campinas, SP, 1990. 
[13] R.W. Jackson e D.M. Pozar, "Full-Wave Analysis of Microstrip OpenEnd and Gap Discontinuities", IEEE Transactions on Microwave Theory and Techniques, vol. MTT-33, 1985, pp. 1036-1042.

[14] F.C. Silva, A.J. Giarola, S.B.A. Fonseca e A.J.M. Soares, "Effect of a Dielectrical Cover in a Microstripline on a Circular Cylindrical Substrate", IEEE Antennas and Propagation Symposium Digest, Dallas, Estados Unidos, 1990, pp. 508-511.

[15] W.L. Stutzman e G.A. Thiele, "Antenna Theory and Design", Wiley, New York, 1981.

[16] R.E. Collin, "Field Theory of Guided Waves", McGraw-Hill Book Company, New York, 1960.

[17] I.E. Rana e N.G. Alexopoulos, "Current Distribution and Input Impedance of Printed Dipoles", IEEE Transactions on Antennas and Propagation, vol. AP-29, 1981, pp. 99-105.

[18] A.J.M. Soares, S.B.A. Fonseca e A.J. Giarola, "The Effect of a Dielectric Cover on the Current Distribution and Input Impedance of Printed Dipoles", IEEE Transactions on Antennas and Propagation, vol. AP-32, 1984, pp. 1149-1153.

[19] J.R. Mosig, "Les Structures Microruban: Analyse Moyen des Équations Intégrales", Tese de Doutorado no 511(1983), Departamento de Eletricidade da Escola Politécnica Federal de Lausanne, Suiça, 1984.

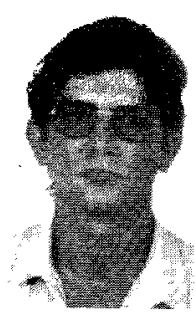

FRANKLIN DA COSTA SILVA nasceu em Ingal, MG, em 1957. Graduou-se em engenharia elétrica pelo Instituto Nacional de Telecomunicações (INATEL) em Santa Rita do Sapucal, MG, em 1981, recebeu o título de Mestre em engenharia elétrica pela Universidade de Brasilia em 1986 e o título de Doutor em engenharia elétrica pela Universidade Estadual de Campinas, SP, em 1990. Seu principal interesse é a utilização de métodos numéricos na solução de problemas de teoria eletromagnética, com ênfase no método dos momentos aplicado à análise de antenas. No momento ele se encontra como Pesquisador Associado e Professor Substituto na Universidade de Brasflia. 


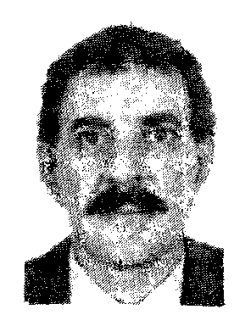

SÉRGIO BARROSO DE ASSIS FONSECA nasceu em Belo Horizonte, MG, em 1944. Graduou-se em engenharia elétrica pela Pontiff́cia Universidade Católica de Minas Gerais (PUC/MG), recebeu o título de Mestre em engenharia elétrica pela Pontiff́cia Universidade Católica do Rio de Janeiro (PUC/RJ) e o tltulo de Doutor em engenharia elétrica pela Universidade Estadual de Campinas em 1982. Ele é no momento Professor Adjunto e Diretor da Faculdade de Tecnologia da Universidade de Brasillia.

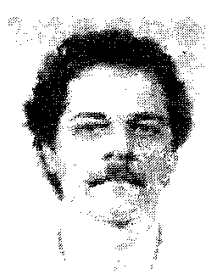

ANTONIO JOSÉ MARTINS SOARES nasceu em Belém, Pará, em 1957. Ele se formou Engenheiro Eletricista pela Universidade Federal do Pará em 1980. E concluiu o Mestrado e o Doutorado em Engenharia Elétrica em 1983 e 1986, respectivamente, na Universidade Estadual de Campinas. Atualmente ele é Professor Adjunto na Universidade de Brasflia, onde trabalha em pesquisas nas áreas de antenas e propagação, com ênfase no uso do método dos momentos para a solução de problemas eletromagnéticos.

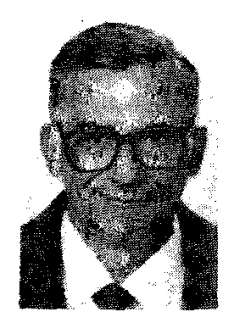

ATTÍlIO JOSÉ GIAROLA nasceu em Jundial, SP, em 1930. Ele se formou como Engenheiro Mecânico e Eletricista pela Escola Politécnica da USP, em 1954. Obteve os graus de mestre e doutor pela Universidade de Washington, Seattle, WA, USA em 1959 e 1963, respectivamente. Antes de obter o grau de doutor ele lecionou por vários ano no ITA, São José dos Campos, $S P$, na Universidade de Seattle e na Universidade de Washington, ambas situadas em Seattle, WA, USA. Em 1962 ele ing ressou na Boeing Company em Seattle, WA, USA e foi responsável por pesquisa em detetores infravermelhos e dispositivos de microondas. Esteve de licença por dois anos da Boeing Co.quando assumiu a posição de Professor Associado no ITA e também foi Professor Visitante na Escola Politécnica da USP. Durante este tempo ele foi o Organizador da Primeira Conferência Nacional em Eletrônica no Brasil e conduziu pesquisa em dispositivos de estado sólido. De 1968 a 1974 ele foi Professor Associado de Engenharia Elétrica na Universidade A \& M do Texas, com responsabilidades de lecionar e conduzir pesquisa em ondas eletromagnéticas, particularmente em suscetibilidade eletromagnética. Em 1975 ele ingressou na Universidade Estadual de Campinas (UNICAMP) oride ele tem conduzido pesquisa em dispositivos de microonda e ópticos e em antenas. Ele foi o Coordenador Geral das Faculdades da UNICAMP de 1980 a 1982 e - Coordenador Geral dos Cursos de Pós-Graduação dessa Uni- 
versidade de 1975 a 1986. Dr. Giarola fol Fresidente da Socieda -

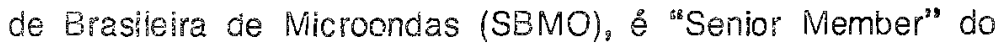
IEEE a membro do Eta Kappa $\mathrm{Nu}$ e do Sigma $\mathrm{Xi}_{\text {. }}$ 